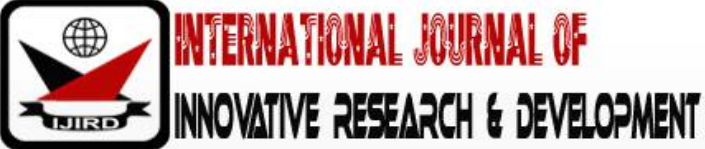

ISSN 2278 - 0211 (Online)

\section{Effect of Imazethapyr 10\% SL as Post Emergence Harbicide on the Growth and Yield of Groundnut (Arachis Hypogaea L.) in Mubi Adamawa State, Nigeria}

Timon David
Lecturer, Department of Botany, Adamawa State University, Mubi, Nigeria
Yusuf Comfort Sankem
Senior Lecturer, Department of Botany, Adamawa State University, Mubi, Nigeria
Batta Kucheli
Lecturer, Department of Botany, Adamawa State University, Mubi, Nigeria
Zakawa Ndale Ngida
Lecturer, Department of Botany, Adamawa State University, Mubi, Nigeria
Waja Samuel
Lecturer, Department of Biological Science Technology,
Federal Polytechnic, Mubi, Nigeria
Mohammed Adamu
Lecturer, Department of Biology Education,
Adamawa State College of Education Hong, Nigeria
Akabia J. J.
Student, Department of Botany, Adamawa State University, Mubi, Nigeria

\begin{abstract}
:
A field experiment was carried out at the Adamawa state university Department of Crop Science Teaching and Research Farm during the rainy season of 2019 ,to Evaluate the Effect of Imazethapyr 10\% SL as post Emergence Herbicide on the Growth and Yield of Groundnut with the objective of selecting the minimum concentration of the herbicides that can control weeds without negatively affecting the growth and yield of groundnut. The treatments consisted of the following: $T_{1}=75 \mathrm{ml}, T_{2}=100 \mathrm{ml}, T_{3}=125 \mathrm{ml}, T_{4}=150 \mathrm{ml}, T_{5}=175 \mathrm{ml}, T_{6}=200 \mathrm{ml}, T_{7}=225 \mathrm{ml}, T_{8}=250 \mathrm{ml}$, $T_{9}=275 \mathrm{ml}$ of imazethapyr dissolve in 16 liter sprayer while $T_{10}=$ Control (hoe weeding). The experiment was laid out in a Randomized Complete Block Design (RCBD) in three replicates, imazethapyr applied 35 days after sowing when the weeds were actively growing. Date collected was subjected to Analysis of Variance (ANOVA), significant means where separated using Duncan Multiple Range Test (DMRT) at $P \leq 0.05$. The results shows significant difference across the levels of the herbicides for most of the growth character, the plot treated with $T_{7}(225 \mathrm{ml} / 16 \mathrm{l}$ sprayer) performed poorly while the control $T_{10}$ (manual weeding) recorded higher growth rate at 2, 4, 6, and 8 Weeks After Treatment Application, higher number of leaves at 2, 4, 6, and 8, higher number of nodes 2, 4, 6, and 8. T, produces more number of immature pod at harvest (9.33) and fewer root nodules per plant (25.00) compared with the other treatments and the control. The yield character such as yield per plot $(\mathrm{kg})$, pod yield in $\mathrm{kg} / \mathrm{ha}$ and harvest index does not vary across the treatments and the control. Significant positive correlation was found between Pod yield in $\mathrm{kg} / \mathrm{ha}$ with biological yield per plot $\left(r=0.4608^{* *}\right)$, pod yield per plot $\left(r=0.7220^{* *}\right)$, Harvest index $\left(r=0.8373^{* *}\right)$, Number of matured pod per plot $\left(r=0.6795^{* *}\right)$, number of pod per plot $\left(r=0.6795^{* *}\right)$, Number of leaves at two weeks ( $\left.r=0.4173^{* *}\right)$, number of nodes at four weeks $\left(r=0.4018^{*}\right)$, plant height at two weeks $\left(r=0.3772^{*}\right)$ and plant height at four weeks $\left(0.4608^{* *}\right)$. Suggest that improvement in those characters will help enhance the yield of groundnut. It can be concluded that application of imazethapyr 10\% SL at the rate of 75-100ml/16 liter sprayer applied 35 days after sowing is recommended for managing complex weed flora and obtaining higher seed yield of groundnut in Mubi, and can serve as a substitute for manual weeding which is time consuming, laborious and expensive.
\end{abstract}

Keywords: Imazethapyr, post emergence, herbicide, growth, yield, groundnut

\section{Introduction}

Groundnut (Arachis hypogaea L.) is a grain legume and oilseed, which is widely cultivated in tropical and subtropical regions. It has a key role in human nutrition. In Africa and Asia, more peanut is grown than any other grain legume (including soy bean) [1]. 
Groundnuts in Nigeria are grown in commercial quantities mostly for the extraction of oil which is used in cooking, the cake which is the by-product of the oil extracted nut is used as an animal feed and also in the production of peanut flour. Apart from extracting the oil content of the nuts, it is also commonly used as a snack and can be boiled, roasted, fried or crushed into candies, and kuli-kuli. Most times it is also used in place of Egusi to make a soup called 'groundnut soup', especially when dried and grounded [2]. Groundnut improves soil fertility through nitrogen fixation, with the help of rhizobium in the root nodules.

China, India, Nigeria, USA and Myanmar are the leading groundnut producing countries in the world. Developing countries in Asia, Africa and South America account for over 97\% of world groundnut area and 95\% of total production. However, the productivity of Asia (2217 kg ha-1) and Africa (929 $\mathrm{kg} \mathrm{ha}^{-1}$ ) is very poor as compared to Americas (3632 kg ha $^{-1}$ ) [3]. Reasons attributed to low productivity of groundnut in Nigeria includes various factors such as non availability of improved seeds, inefficient fertilizer use, weed infestation, shortage of irrigation water, drought, seasonal variation of rainfall, inadequate research efforts and inefficient extension services [4].

Weeds reduce yield by competing with the groundnut plants for resources such as sunlight, space, moisture and nutrients throughout the growing season [5].

Herbicides and hand weeding significantly brought down the nutrient removal by weeds and enhanced the uptake of nutrient by groundnut crop [6]. [7] opined that hand weeding twice significantly increased the kernal yield of groundnut upto 2.42 times than unweeded control. [8] reported that the farmers practice of hand weeding twice on 20 and 40 days after sowing (DAS) resulted in lower weed dry matter and higher pod yield (1496kgha-1)in rabi ground nut compared with application of pendimethalinat $1.0 \mathrm{~kg} \mathrm{ha}^{-1}\left(714 \mathrm{~kg} \mathrm{ha}^{-1}\right)$. [9] reported that hand weeding twice at 2nd and 4 th weeks after sowing was effective to control weeds and recommended to improve vegetative growth of groundnut. But this cultural method is time consuming, energy sapping and costly.

[10] An important and relatively less expensive control option is chemical weed control, which involves the use of different herbicides applied as PRE and POST-emergence of crops. [11] estimated that non-use of herbicides for weed control in crops could increase cost of production by $20 \%$. The use of herbicide for weed control in groundnut can significantly reduce the need of labour for hand weeding thereby lowering the cost of production. On per hectare estimation, herbicide could replace approximately the need for 10 laborers in weed control.

The use of herbicides is therefore often considered an effective alternative to hand weeding. This alternative as observed by [12] is often applicable to large hectares of farm land where hand weeding may not be feasible due to labour and other logistic constraints. The increasing scarcity and high cost of labour for manual weeding necessary to achieve adequate yields of groundnut in Nigeria have led to growing interest in herbicides [13].

The most effective way to tackle the problem of weeds on groundnut field is to apply a post emergence herbicide like imazethapyr which will eliminate the weeds without having an adverse effect on the groundnut itself. Imazethapyr is the first herbicide registered in peanut to provide both post-emergence and residual control of many problem weeds [14]. [15] reported that post emergence application Imazethapyr was very effective in controlling weeds in lentil. The critical period of crop-weed competition was found to be 4 to 8 weeks after sowing [16]. Thus, in case of groundnut, early removal of weeds before flowering and during pegging is important [17]. [18] reported that, chemical control of weeds forms an excellent alternative to manual weeding.

Therefore this research is design to determine the effect of imazethapyr $10 \%$ SL as post emergence herbicide on the growth and yield of groundnut with the objective of selecting the minimum concentration of imazethapyr that can control the emergent grasses without affecting the growth and yield of groundnut.

\section{Materials and Methods}

\subsection{Description of the Study Area}

The research was carried out at the Faculty of Agriculture Department of Crop Science Teaching and Research Farm, Adamawa State University Mubi, during the 2019 rainy season. Latitude $10^{\circ} 10^{\prime}$ and longitude $10^{\circ} 30^{\prime}$ north of the equator and between longitude $13^{0} 10^{\prime}$ and $13^{\circ} 30^{\prime}$ East of Greenwich meridian and at an altitude of $696 \mathrm{~m}$ above sea level.

\subsection{Sources of Seed}

The seeds for the experiment were purchased from local farmers in Mubi and it was tested for viability by floatation method before planting.

\subsection{Land Preparation}

The land was disc ploughed and harrowed to a fine tilt. The main plot size was $7 \times 24 \mathrm{~m}^{2}$ which was divided into 2 $\mathrm{x} 2 \mathrm{~m}^{2}$ subplots and a pathway of $0.5 \mathrm{~m}$ between subplots for easy movement of water.

\subsection{Sowing}

Two seeds were sown two per hole at a depth of $2 \mathrm{~cm}$ and covered with soil at a spacing of $30 \mathrm{~cm}$ intra row spacing and $40 \mathrm{~cm}$ inter row spacing, which was later tinned to one per stand at 2 weeks after sowing.

\subsection{Treatments and Experimental Design}

The treatments consisted of different levels of imazethapyr $10 \%$ SL as follows:

$\mathrm{T}_{1}=75 \mathrm{ml}, \mathrm{T}_{2}=100 \mathrm{ml}, \mathrm{T}_{3}=125 \mathrm{ml}, \mathrm{T}_{4}=150 \mathrm{ml}, \mathrm{T}_{5}=175 \mathrm{ml}, \mathrm{T}_{6}=200 \mathrm{ml}, \mathrm{T}_{7}=225 \mathrm{ml}, \mathrm{T}_{8}=250 \mathrm{ml}, \mathrm{T}_{9}=275 \mathrm{ml} \quad$ of imazethapyr 10\% SL dissolve in 16 liter of water and sprayed using 16 Liter knapsack sprayer while $\mathrm{T}_{10}=$ Control $($ Hoe 
weeding).Five (5) weeks after sowing when the grasses have emerged and are actively growing, the treatments were applied across the experimental plot in a Randomized Complete Block Design (RCBD) in three replicates.

\subsection{Data Collection}

Data was collected from five randomly selected plants from each treatment on the following Growth characters: Plant height, Number of leaves, Number of nodes at 2, 4, 6 and 8 weeks after treatments application. While the yield character observed were: Number of immature pods per plant, Number of immature pods per plot, Number of matured pods per plot, Number of matured pods per plant, Number of pods per plant, Number of pods per plot, Number of root nodules per plant, 1000 seed weight, Biological yield per plot, Grain yield per plot, Grain yield $\mathrm{kg} / \mathrm{ha}$ is calculated using the formula Grainyield $(\mathrm{kg} / \mathrm{ha})=\frac{\text { Grainyield }(\mathrm{kg})}{\text { Areaofsubplot }(\mathrm{m} 2)} \times 10,000 \mathrm{~m}^{2}$ while Harvest index is calculated using the formula

$$
\text { Harvestindex }(H I)=\frac{\text { Economicyield }(\mathrm{kg})}{\text { BiologicalYield }(\mathrm{kg})}
$$

\subsection{Data Analyses}

The data collected was subjected to analysis of variance (ANOVA) and correlation computed using Statistical Package for Scientist and Engineers (SPSE). Significant means were separated using Duncan Multiple Range Test (DMRT) at $\mathrm{P} \leq 0.05$.

\section{Results}

\subsection{Physical and Chemical Properties of the Soil from Experimental Site}

The soil texture of the experimental site is sandy clay with $70 \%$ sand and $21 \%$ clay with pH 8.0 which is slightly basic. It is deficient in major nutrients like; nitrogen $0.048 \%$, phosphorus $0.035 \%$, and potassium $0.9 \%$. It has high percentage of zinc $43.54 \mathrm{mg} / \mathrm{kg}$ (Table 1 ).

\subsection{Effect of Imazethapyr 10\% SL on Plant Height and Number of Leaves at Various Weeks of Herbicide Application}

The Analysis of Variance (ANOVA) showed significant difference at $\mathrm{P}<0.05$ for plant height at 2, 4, 6 and 8 weeks after treatment application (WATA) with $\mathrm{T}_{10}=$ hoe weeding attaining a height of $27.27 \mathrm{~cm}, 29.23 \mathrm{~cm}, 31.00 \mathrm{~cm}$ and $33.93 \mathrm{~cm}$ respectively. While $\left(\mathrm{T}_{7}\right)$ recorded the least plant height of $14.06 \mathrm{~cm}, 16.67 \mathrm{~cm}$, and $23.80 \mathrm{~cm}$ at 2,4 and 8 WATA respectively, while at 6 WATA $\left(\mathrm{T}_{6}\right)$ recorded the least plant height of $20.33 \mathrm{~cm}$ (Table 2).

The Analysis of Variance (ANOVA) also revealed significant difference at $\mathrm{P}<0.05$ for number of leaves at 2, 4, 6 and 8 WATA the control $\left(\mathrm{T}_{10}\right)$ hoe weeding recorded the highest number of leaves of 265.33, 285.33, 308.00 respectively while at 8 WATA, $\left(\mathrm{T}_{5}\right)$ recorded the highest number of leaves of 397.00 , the lowest number of leaves was recorded in $\mathrm{T}_{7}$ at 2, 4, 6 and 8 WATA as $130.33,158.00,221.33$ and 320.33 respectively. The number of leaves produced by the other treatments in the respective weeks do not differ significantly (Table 3).

\subsection{Effect of Imazethapyr 10\% SL on Number of Nodes, Matured Pods Per Plant, Matured Pods Per Plot, Number of Pods Per Plant and Number of Pods Per Plot}

The Analysis of Variance (ANOVA) revealed significant difference at $\mathrm{P}<0.05$ for number of nodes at 2, 4, 6 and 8 WATA, the control $\left(\mathrm{T}_{10}\right)$ hoe weeding recorded the highest number of nodes of $67.67,72.67$ and 78.67 respectively, while at 8 WATA, while $T_{6}$ recorded the highest number of nodes of 97.33 , the lowest number of nodes was recorded in $\mathrm{T}_{7}$ at $2,4,6$ and 8 WATA as $32.33,38.33,52.67,78.33$ respectively. The number of nodes produced by the other treatments in the respective weeks do not differ significantly (Table 4).

ANOVA revealed no significant difference at $\mathrm{P}<0.05$ for number of matured pods per plant, matured pods per plot, number of pod per plant and number of pod per plot. (Table 5)

Effect of imazethapyr 10\% SL on Number of Immature pods per plant, immature pods per plot, root nodules per plant and one thousand seed weight, Biological yield per plot, Economic yield per plot,Harvest index andGrain yield kgha-1 and ANOVA revealed significant difference across the different treatments and the control with $\mathrm{T}_{9}$ yielded the highest number of immature pod per plant 9.33 while $\mathrm{T}_{4}$ yielded the lowest number of immature pod per plant 6.33. There was also variation in the number of root nodules per plant with the control $\mathrm{T}_{10}$ recorded the highest number of root nodules per plant 67.67 while $\mathrm{T}_{9}$ recorded the lowest root nodules per plant of 25.00. One thousand seed weight also showed significant different across the various treatments with $\mathrm{T}_{4}$ recorded the highest weight of $466.67 \mathrm{~g}$ while the lowest was recorded in $\mathrm{T}_{7} 412.33 \mathrm{~g}$ (Table 6).

ANOVA showed no significant difference at $\mathrm{P}<0.05$ for biological yield per plot with $\mathrm{T}_{3}$ yielded the highest biological yield per plot of $0.93 \mathrm{~kg}$, while the least biological yield was produced by $\mathrm{T}_{1}=0.63 \mathrm{~kg}$. Economic yield per plot, harvest index and pod yield in $\mathrm{kg} / \mathrm{ha}$ do not show significant difference across the various treatments and the control (Table 7).

\subsection{Pearson Correlation between Pod Yield In $\mathrm{Kg} / \mathrm{Ha}$ and Other Phenotypic Traits}

There was high significant correlation between Pod yield in $\mathrm{kg} / \mathrm{ha}$ with biological yield per plot ( $\left.\mathrm{r}=0.4608^{* *}\right)$, pod yield per plot $\left(\mathrm{r}=0.7220^{* *}\right)$, Harvest index $\left(\mathrm{r}=0.8373^{* *}\right)$, Number of matured pod per plot $\left(\mathrm{r}=0.6795^{* *}\right)$, number of pod per plot $\left(\mathrm{r}=0.6795^{* *}\right)$, Number of leaves at two weeks $\left(\mathrm{r}=0.4173^{* *}\right)$, number of nodes at four weeks $\left(\mathrm{r}=0.4018^{*}\right)$, plant height at two weeks $\left(\mathrm{r}=0.3772^{*}\right)$ and plant height at four weeks $\left(0.4608^{* *}\right)$ (Table 8). 


\begin{tabular}{|lc|}
\hline Parameter & valued obtained \\
\hline Sand & $70 \%$ \\
Silt & $9 \%$ \\
Clay & $21 \%$ \\
Chemical properties parameter & valued obtained \\
Soil PH & 8.00 \\
Sodium $(\mathrm{Na}) \%$ & 0.13 \\
Magnesium $(\mathrm{mg}) \%$ & 1.12 \\
Total potassium $(\mathrm{K}) \%$ & 0.90 \\
Available calcium $(\mathrm{Ca}) \%$ & 1.15 \\
Copper $(\mathrm{cu}) \mathrm{mg} / \mathrm{kg}$ & 12.66 \\
Iron (fe) $\mathrm{mg} / \mathrm{kg}$ & 17.13 \\
Zinc (zn) $\mathrm{mg} / \mathrm{kg}$ & 43.54 \\
Phosphorus $(\mathrm{p}) \%$ & 0.04 \\
Nitrogen $(\mathrm{N}) \%$ & 0.05 \\
Carbon $(\mathrm{C}) \%$ & 1.18 \\
Conductivity Ng/cm & 875.15 \\
\hline
\end{tabular}

Table 1: Physical and Chemical Properties of the Soil from Experimental Site

\begin{tabular}{|c|c|c|c|c|c|c|c|c|c|}
\hline \multirow[b]{2}{*}{ Code } & \multirow[b]{2}{*}{$\begin{array}{c}\text { Herbicide } \\
\text { Concentra } \\
\text { tions } \\
\text { (ml/16 } \\
\text { Liter } \\
\text { sprayer) }\end{array}$} & \multicolumn{8}{|c|}{ Weeks after Herbicide Application } \\
\hline & & PLH2Wks & PLH4Wks & PLH6Wks & PLH8Wks & NL2wks & NL4wks & NL6wks & NL8wks \\
\hline $\mathrm{T}_{1}$ & 75 & $17.40^{\mathrm{b}}$ & $21.33^{b}$ & $25.00^{\mathrm{b}}$ & $28.40^{\mathrm{b}}$ & $162.67 \mathrm{bc}$ & $192.67 \mathrm{~b}$ & $250.00 \mathrm{ab}$ & $310.33^{b}$ \\
\hline $\mathrm{T}_{2}$ & 100 & $19.07 \mathrm{~b}$ & $21.17 \mathrm{~b}$ & 23.17 bc & $24.40^{\mathrm{c}}$ & $226.00^{\mathrm{ab}}$ & $247.00^{a b}$ & 276.00 ab & 293.33 b \\
\hline $\mathrm{T}_{3}$ & 125 & $18.67 \mathrm{~b}$ & $21.17 \mathrm{~b}$ & $23.23 \mathrm{bc}$ & $25.00 \mathrm{bc}$ & $220.33^{\mathrm{abc}}$ & $246.00 \mathrm{ab}$ & 301.67 a & $338.67 \mathrm{ab}$ \\
\hline $\mathrm{T}_{4}$ & 150 & $17.47 \mathrm{bc}$ & $20.33 \mathrm{bc}$ & $23.00 \mathrm{bc}$ & $25.13 \mathrm{bc}$ & $177.33 \mathrm{abc}$ & $197.00 \mathrm{ab}$ & $253.33 \mathrm{ab}$ & $324.00 \mathrm{ab}$ \\
\hline $\mathrm{T}_{5}$ & 175 & $16.80 \mathrm{bc}$ & $20.00 \mathrm{bc}$ & $23.77 \mathrm{bc}$ & $26.53 \mathrm{bc}$ & $176.33 \mathrm{abc}$ & $209.67 \mathrm{ab}$ & $269.67 \mathrm{ab}$ & $392.00^{a}$ \\
\hline $\mathrm{T}_{6}$ & 200 & $14.33 \mathrm{bc}$ & $17.00 \mathrm{bc}$ & $20.33^{c}$ & $24.40^{c}$ & $151.00^{\mathrm{bc}}$ & $222.67 \mathrm{ab}$ & $252.33^{\mathrm{ab}}$ & $274.67^{b}$ \\
\hline $\mathrm{T}_{7}$ & 225 & $14.06^{c}$ & $16.67 \mathrm{c}$ & $20.50^{c}$ & $23.80^{c}$ & $130.33^{c}$ & $158.00^{b}$ & $221.33^{b}$ & $320.33^{b}$ \\
\hline $\mathrm{T}_{8}$ & 250 & $14.33 \mathrm{bc}$ & 18.67 bc & 21.35 bc & 24.67 bc & $161.33 \mathrm{bc}$ & $220.67 \mathrm{ab}$ & $256.67 \mathrm{ab}$ & $321.67 \mathrm{~b}$ \\
\hline $\mathrm{T}_{9}$ & 275 & $16.07 \mathrm{bc}$ & $19.67 \mathrm{bc}$ & $22.00 \mathrm{bc}$ & $24.67 \mathrm{bc}$ & $160.00^{\mathrm{bc}}$ & $210.67 \mathrm{ab}$ & $286.33 \mathrm{ab}$ & $336.33 \mathrm{ab}$ \\
\hline $\mathrm{T}_{10}$ & $\begin{array}{c}\text { (Hoe } \\
\text { weeding) }\end{array}$ & $27.27^{a}$ & $29.23^{a}$ & $31.00^{a}$ & $33.93^{a}$ & $265.33^{a}$ & $285.33^{a}$ & 208.00 a & $333.00 \mathrm{ab}$ \\
\hline SE \pm & & 2.33 & 2.09 & 1.78 & 1.86 & 45.32 & 44.28 & 37.22 & 35.32 \\
\hline
\end{tabular}

Table 2: Effect of Imazethapyr 10\% SL on Some Growth Parameters of Groundnut

Mean followed by the same superscript within the same column and treatment are not significantly different at $\mathrm{P}$ $\leq 0.05$ (DMRT). ${ }^{*}=$ Significant at $\mathrm{P} \leq 0.05,{ }^{* *}=$ Significant at $\mathrm{P} \leq 0.01, \mathrm{NS}=$ Not significant $\mathrm{PLH}=\mathrm{Plant}$ Height, NL= number of leaves, wks $=$ Weeks after Treatment Application

\begin{tabular}{|c|c|c|c|c|c|c|c|c|c|}
\hline \multirow[b]{2}{*}{ Code } & \multirow[b]{2}{*}{$\begin{array}{c}\text { Herbicide } \\
\text { Concentrat } \\
\text { ions } \\
\text { (ml/16 } \\
\text { Liter } \\
\text { sprayer) }\end{array}$} & \multicolumn{8}{|c|}{ Weeks after Herbicide Application } \\
\hline & & NN2Wks & NN4Wks & NN6Wks & NN8Wks & MPPplant & MPP plot & NPP plant & NPPplot \\
\hline $\mathrm{T}_{1}$ & 75 & $44.67 \mathrm{bc}$ & $50.00^{\mathrm{abc}}$ & 64.67abc & $77.67 \mathrm{bc}$ & $8.00^{\mathrm{a}}$ & 147.67 a & $13.00^{\mathrm{a}}$ & $202.33^{a}$ \\
\hline $\mathrm{T}_{2}$ & 100 & $56.33^{\mathrm{ab}}$ & $63.00^{\mathrm{ab}}$ & $68.33 \mathrm{abc}$ & $73.33 \mathrm{bc}$ & 10.67 a & 151.67 a & $19.33^{a}$ & 234.67 a \\
\hline $\mathrm{T}_{3}$ & 125 & $44.33^{\mathrm{abc}}$ & $65.00^{\mathrm{ab}}$ & 73.67ab & $84.33^{\mathrm{abc}}$ & $10.33^{a}$ & 165.67 a & 17.67 a & $266.33^{a}$ \\
\hline $\mathrm{T}_{4}$ & 150 & $40.00^{\mathrm{abc}}$ & $54.00^{\mathrm{abc}}$ & $64.00^{\mathrm{abc}}$ & $81.33^{\mathrm{abc}}$ & $11.00^{\mathrm{a}}$ & $148.33^{a}$ & $15.33^{a}$ & $232.00 \mathrm{a}$ \\
\hline $\mathrm{T}_{5}$ & 175 & $44.00 \mathrm{bc}$ & $53.00 \mathrm{abc}$ & $75.00^{\mathrm{ab}}$ & $68.67 \mathrm{c}$ & $8.67 \mathrm{a}$ & 103.33 a & $16.67 \mathrm{a}$ & 207.33 a \\
\hline $\mathrm{T}_{6}$ & 200 & 37.67 c & 44.67 bc & 58.00 bc & $97.33^{\mathrm{a}}$ & $8.67^{a}$ & $77.00^{a}$ & $12.33^{\mathrm{a}}$ & $163.33^{a}$ \\
\hline $\mathrm{T}_{7}$ & 225 & $32.33^{\mathrm{abc}}$ & 38.33abc & $52.67 \mathrm{c}$ & 78.33 bc & $9.33^{a}$ & $114.00^{a}$ & $14.67 \mathrm{a}$ & $181.67 \mathrm{a}$ \\
\hline $\mathrm{T}_{8}$ & 250 & $40.00^{a b c}$ & $54.33^{\mathrm{abc}}$ & 69.00abc & 80.67 bc & $10.33^{a}$ & $167.00 \mathrm{a}$ & $17.00^{\mathrm{a}}$ & 233.67 a \\
\hline $\mathrm{T}_{9}$ & 275 & $40.00^{\mathrm{a}}$ & $51.33^{a b c}$ & $63.67 \mathrm{abc}$ & $84.33^{\mathrm{abc}}$ & $8.33^{a}$ & $92.33^{a}$ & $13.67 \mathrm{a}$ & $202.67^{a}$ \\
\hline $\mathrm{T}_{10}$ & $\begin{array}{c}\text { (Hoe } \\
\text { weeding) }\end{array}$ & 67.67 & $72.67^{a}$ & $78.67 \mathrm{a}$ & 85.67ab & 10.67 a & 155.00 a & $19.33^{a}$ & $241.33^{a}$ \\
\hline $\mathrm{SE} \pm$ & & 11.34 & 11.82 & 9.28 & 7.88 & 1.78 & 64.82 & 3.71 & 65.52 \\
\hline
\end{tabular}

Table 3: Effect of Imazethapyr 10\% SL on Some Groundnut Attributes 
Mean followed by the same superscript within the same column and treatment are not significantly different at $\mathrm{P}$ $\leq 0.05$ (DMRT). ${ }^{*}=$ Significant at $\mathrm{P} \leq 0.05,{ }^{* *}=$ Significant at $\mathrm{P} \leq 0.01$, NS=Not significant, NN=number of nodes, MPPplant= Matured pod per plant, $\mathrm{MPP}_{\text {plot }}$ Matured pod per plot, NPP plant= Number of pod per plant, NPP plot= Number of pod per plot

\begin{tabular}{|c|c|c|c|c|c|c|c|c|c|}
\hline \multirow[b]{2}{*}{ Code } & \multirow[b]{2}{*}{$\begin{array}{c}\text { Herbicide } \\
\text { Concentrations } \\
\text { (ml/16 Liter } \\
\text { sprayer) }\end{array}$} & \multicolumn{8}{|c|}{ Weeks after Herbicide Application } \\
\hline & & IMPP plant & IMPP $_{\text {plot }}$ & $\mathbf{R N P}_{\text {plant }}$ & TSW (g) & BYP plot (kg) & SYP plot (kg) & HI & SY(kg ha-1) \\
\hline $\mathrm{T}_{1}$ & 75 & $7.00^{\mathrm{ab}}$ & $54.67 \mathrm{a}$ & $29.00^{\mathrm{bc}}$ & $456.67 \mathrm{ab}$ & $0.63^{b}$ & $0.07 \mathrm{a}$ & $0.28^{\mathrm{a}}$ & 466.67 a \\
\hline $\mathrm{T}_{2}$ & 100 & $7.33^{\mathrm{ab}}$ & $83.00^{a}$ & $42.33 \mathrm{bc}$ & $438.67 \mathrm{ab}$ & $0.83^{\mathrm{ab}}$ & $0.13^{a}$ & $0.16^{\mathrm{a}}$ & $375.00^{a}$ \\
\hline $\mathrm{T}_{3}$ & 125 & $7.33^{a b}$ & 101.00 a & $34.33 \mathrm{bc}$ & $451.33^{\mathrm{ab}}$ & $0.93^{a}$ & $0.10^{a}$ & $0.24^{a}$ & 451.67 a \\
\hline $\mathrm{T}_{4}$ & 150 & $6.33^{b}$ & 83.67 a & $40.00 \mathrm{bc}$ & $466.67 \mathrm{a}$ & $0.77 \mathrm{ab}$ & $0.07 \mathrm{a}$ & 0.27 a & 533.33 a \\
\hline $\mathrm{T}_{5}$ & 175 & $8.67 \mathrm{ab}$ & 104.00 a & $29.00 \mathrm{bc}$ & $457.67 \mathrm{ab}$ & $0.83^{\mathrm{ab}}$ & $0.07 \mathrm{a}$ & $0.21^{\mathrm{a}}$ & 466.67 a \\
\hline $\mathrm{T}_{6}$ & 200 & $8.33^{\mathrm{ab}}$ & 59.67 a & $34.67 \mathrm{bc}$ & $450.33^{\mathrm{ab}}$ & $0.67 \mathrm{ab}$ & $0.07 \mathrm{a}$ & $0.23^{\mathrm{a}}$ & 375.00 a \\
\hline $\mathrm{T}_{7}$ & 225 & $9.00^{a}$ & 67.67 a & $33.33 \mathrm{bc}$ & $412.33^{b}$ & $0.73^{\mathrm{ab}}$ & $0.10^{a}$ & $0.20^{a}$ & 366.67 a \\
\hline $\mathrm{T}_{8}$ & 250 & $9.00^{a}$ & 66.67 a & $45.00^{\mathrm{b}}$ & $429.00^{\mathrm{ab}}$ & $0.83^{\mathrm{ab}}$ & $0.10^{a}$ & $0.24^{\mathrm{a}}$ & $508.33^{a}$ \\
\hline $\mathrm{T}_{9}$ & 275 & $9.33^{a}$ & $110.33^{a}$ & $25.00^{c}$ & $439.67 \mathrm{ab}$ & $0.73^{\mathrm{ab}}$ & $0.07^{a}$ & $0.24^{\mathrm{a}}$ & 441.67 a \\
\hline $\mathrm{T}_{10}$ & (Hoe weeding) & $8.33^{a}$ & $86.33^{a}$ & 67.67 a & 438.00 & $0.77 \mathrm{ab}$ & $0.07 \mathrm{a}$ & $0.28^{a}$ & 533.33 a \\
\hline $\mathrm{SE} \pm$ & & 1.27 & 39.47 & 9.55 & 22.09 & 0.14 & 0.13 & 0.06 & 167.79 \\
\hline
\end{tabular}

Table 4: Effect of Imazethapyr 10\% SL on Some Groundnut Attribute

Mean followed by the same superscript within the same column and treatment are not significantly different at $\mathrm{P}$

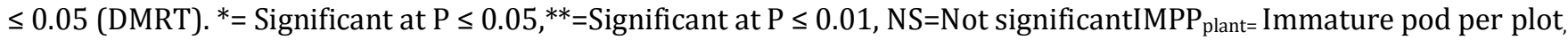
$\mathrm{IMPP}_{\text {plot }}$ Immature pod per plot, $\mathrm{RNP}_{\text {plant= }}$ Root nodules per plant, TSW= One thousand seed weight, BYP $\mathrm{plot}_{(\mathrm{kg})=\mathrm{Biological}}$

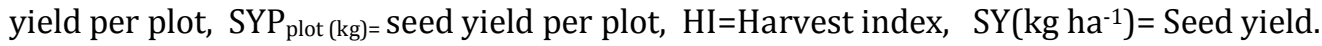

\begin{tabular}{|c|c|c|c|c|c|c|c|c|c|}
\hline & BYP $_{\text {Plot }}$ & SYKg/ha & SYP $_{\text {Plot }}$ & H I & NL8wks & MPP $_{\text {Plot }}$ & $\mathbf{N P P}_{\text {Plot }}$ & IMPP $_{\text {Plot }}$ & $\mathbf{R N P}_{\text {Plot }}$ \\
\hline SY Kg/ha & $0.4608^{* *}$ & & & & & & & & \\
\hline SYP $_{\text {Plot }}$ & $0.6095^{* *}$ & $0.7220^{* *}$ & -------- & & & & & & \\
\hline H I & ---------- & $0.8373^{* *}$ & $0.6606^{* *}$ & & & & & & \\
\hline LA8wks & $0.4624^{* *}$ & ---------- & ---------- & ---------- & ---------- & --------- & ----------- & $0.4072^{*}$ & ------- \\
\hline $\mathrm{MPPP}_{\text {lot }}$ & $0.5687^{* *}$ & $0.6687^{* *}$ & $0.6133^{* *}$ & $0.5064^{* *}$ & 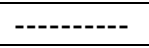 & --------- & --------- & ------- & ------- \\
\hline $\mathrm{NPPP}_{\text {lot }}$ & $0.7653^{* *}$ & $0.6795^{* *}$ & $0.7273^{* *}$ & $0.4982^{* *}$ & $0.7311^{* *}$ & $0.8057^{* *}$ & --------- & 0.8057 & ------- \\
\hline NL2 wks & $0.4784^{* *}$ & $0.4179^{*}$ & $0.3611^{*}$ & ------- & ------ & ------- & & -------- & $0.3563^{*}$ \\
\hline NN2wks & $0.4705^{* *}$ & $0.4294^{*}$ & $0.3709 *$ & ----------- & ---------- & $0.4264^{*}$ & ---------- & ------- & $0.3710^{*}$ \\
\hline NN4wks & ------- & $0.4018^{*}$ & 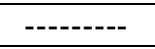 & --------- & 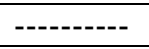 & $0.3819 *$ & (-------- & ------- & $0.3677^{*}$ \\
\hline PLH 2wks & --------- & $0.3772^{*}$ & --------- & $0.3698^{*}$ & --------- & $0.4059 *$ & $0.4085^{*}$ & -------- & $0.4709 * *$ \\
\hline PLH4wks & ------- & $0.3559 *$ & $0.3418^{*}$ & ------- & --------- & ------- & $0.4493^{*}$ & ------- & $0.4514^{* *}$ \\
\hline PLH8wks & -------- & ------ & --------- & -------- & --------- & ------- & $0.3726^{*}$ & ------- & $0.4953^{* *}$ \\
\hline
\end{tabular}

Table 5: Pearson Correlation for Growth and Yield Character in Groundnut

* Significant At $P<0.05$ ** Significant at $P<0.01$ NS = Not Significant

$\mathrm{BIYPP}=$ Biological yield per plot, YI Kg/ha=Pod yield in $\mathrm{Kg} / \mathrm{ha}, \mathrm{HI}=$ Harvest index $\mathrm{NL}=\mathrm{Number}$ of leaves $\mathrm{NPPP}$ lot= number of pod per plot, MPPP ${ }_{\text {lot }}=$ Matured pod per plot, IMPPP ${ }_{\text {lot }}=$ Immature pod per plot, RNPP $_{\text {lnt }}=$ root nodules per plant, $\mathrm{PLH}=$ Plant height, NN=Number of nodes.

\section{Discussion}

There was high significant difference for plant height at 2, 4, 6, and 8 weeks after treatment application, the control (manual weeding) recorded the highest plant height compared with the other treatments while the higher concentrations of herbicides $\mathrm{T} 7=225 \mathrm{ml} / 16$ liter recorded the least plant height. This result therefore suggest that the herbicide concentration up to that level had a detrimental effect on the growth of groundnut by affecting some physiological processes in the plant leading to stunted growth. This result agrees with the findings of [19] who reported that proper weed control was responsible for increase in plant height and dry matter production in groundnut.

Number of leaves and number of nodes showed significant difference across the various treatments and the control, manual weeding also recorded the highest number of leaves and nodes, while the higher concentrations like T7, T8, and T9 recorded the least number of leaves and nodes. This result is not surprising because the toxicity of the herbicides might have tempered with the photosynthetic activity and nutrient assimilation leading to stunted growth and reduced photosynthetic area.

Most of the yield character observed in this research such as Number of matured pod per plot, number of pod per plant, number of pod per plot number of immature pod per plot, pod yield per plot, harvest index and seed yield in $\mathrm{kg} / \mathrm{ha}$ does not vary between the treatments and the control. These results therefore suggest that imazethapyr has effect on the 
growth of plant to certain extent but do not necessarily affect the yield of groundnut as most of the yield character were not affected by the application of imazethapyr. The result is contrary to the findings of [20] on groundnut who reported that manual weeding of groundnut gives the highest yield.

Number root nodules per plant, one thousand seed weight and biological yield per plot varies significantly across the various herbicides treatments with the higher concentrations yielded more immature pod per plot, fewer root nodules, lighter one thousand seed weight and yielded the least biological yield per plot. Suggest that those characters were seriously affected by the application of imazethapyr.

Significant positive correlation was observed between pod yield in $\mathrm{kg} / \mathrm{ha}$ with biological yield per plot, pod yield per plot, Harvest index, Number of matured pod per plant, number of pod per plot, Number of leaves at two weeks, number of nodes at four weeks, plant height at two weeks and plant height at four weeks. Suggest that improvement in any of this character will help enhance the yield of groundnut. Therefore these characters should be given more priority when planning hybridization aim at improving the yield of groundnut. The results agree with the finding of several research. [21] reported that pod yield per plant exhibited significant positive correlation with kernel yield per plant, number of kernel per plant, hundred kernel weight [22] observed similar association of pod yield per plant with other yield contributing characters, [23] also reported significant positive association for pod yield per plant with kernel yield and 100 seed weight.

\section{Conclusion}

Imazethapyr 10\% SL can perfectly substitute manual weeding in groundnut production since the performance in most of yield character do not differ significantly across the various treatments and the control. Therefore application of imazethapyr 10\% SL at higher concentrations of between 250-275 ml per 16 liter sprayer applied once at five (5) weeks after sowing can perfectly control the weeds but will adversely affect the growth character of groundnut. But for the yield characters, it has no effect significant effect on them. Therefore the application of lower rate of $75-100 \mathrm{ml} / 16$ liter applied at 5 weeks after sowing can completely control the emergent weeds without affecting the yield of groundnut as most of the yield character were not affected by therefore this herbicide can be a substitute to manual weeding which is laborious, time consuming and cost effective. Significant positive correlation between pod yield in $\mathrm{kg} / \mathrm{ha}$ with biological yield per plot, pod yield per plot, Harvest index, Number of matured pod per plant, number of pod per plot, Number of leaves at two weeks, number of nodes at four weeks, plant height at two weeks and plant height at four weeks. Suggest that improvement in any of this character will help enhance the yield of groundnut. Therefore these characters should be given more priority when planning hybridization aim at improving the yield of groundnut.

\section{Recommendations}

- I recommend the use of imazethapyr $10 \%$ SL at the rate of $75-100 \mathrm{ml} / 16$ Litre spray at 5 weeks after sowing for the control of weeds, and enhance productivity in groundnut.

- Further research to be carried out over locations and seasons for stability

- Lower levels of Imazethapyr $10 \%$ SL to be tested on groundnut.

- Imazethapyr $10 \%$ SL should be tested on other leguminous crops such as soya bean, bambara nut, cowpea etc

\section{References}

i. FAO. (2015). Food and Agriculture Organization Corporate Statistical Database (FAOSTAT), http://faostat3.fao.org/home/Accessed 24th January, 2015

ii. Anon (2016). Groundnut Producing States In Nigeria and Its Commercial Uses https://www.finelib.com/about/nigeria-cash-crops/groundnut-producing-states-in-nigeria-and-itscommerical-uses/90 Accessed 24th March, 2020

iii. FAO. (2014). Statistical Data Base. Food and Agricultural Organization of the United Nations. Retrieved fromhttp://www.fao.org

iv. Ali, S.,Scott, J.F., People, M.B., Schwanke, G.D., and Herridge, D.F. (2002). Nitrogen, yield and economic benefits of summer legumes for wheat production in rainfed Northern Pakistan. Pakistan Journal of Agronomy1:15-19.

v. UpadhyayU.C. (1984). Weed Management in Oilseed Crops. Proceeding Symposium. Oilseed Production, Utilization, Constraints and Opportunities, pp. 491-99.

vi. Yadav S.K. Singh S P. and Bhan V M. (1983). Performance of herbicides for weed control in groundnut. Indian Journal of Weed Science, 15: 58-61.

vii. Meyyappan, M and Kathiresan R.M (2005). Integrated weed management in maize + blackgram intercropping system. Indian Journal of Weed Science37: 209-211.

viii. Madhavi, M. D., Madhusudhan R., Padmavathi D and Reddy C.N(2008). Integrated weed management inmaizegroundnut cropping system in southern Telangana zone of Andra Pradesh. In: Biennial conference on weed management in modern agriculture: Emerging challenges and opportunities. 27-28 February. Organised by ISWS, NRCWS, Jabalpur (M.P) and Rajendra Agricultural University, Pusa (Bihar). P. 125.

ix. Naim A.M., Eldouna, M.A and Abdalla A.E (2010). Effect of weeding frequencies and plant density on the vegetativegrowth characteristic in groundnut (Arachis hypogea L.) in North kordofan of Sudan. 1 (3): 11881192.

x. Akobundu, I.O. (1980). Weed control in cowpea in the humid tropics. Weed Science Journal of Nigeria, 4: 6-1. 
xi. Gianessi, L. P., and Reigner, N. P. (2007). The value of herbicides in United States crop production. Weed Technology, 21(2),559-566.

xii. Adigun, J. A. and Lagoke, S. T. (1994). Chemical weed control in Maize groundnut mixture in Northern Guinea savannah ecological zone of Nigeria. Nigeria Agricultural Journal, 27:2

xiii. Akobundu, I. O. (1982). The role of Conservation Tillage in Weed Management in the Advancing Countries.FAO Plant Production and Protection Paper, 44:23-39).

xiv. Grichar, W.J and Sestak D.C. (2000). Effect of adjuvant on control of nutsedge (Cyperus esculentus and C. rotundus) byimazapic and imazethapyr. Crop Protection 19: 461-465

xv. Guriqbal Singh., Harpreet Kaur, and Veena Khanna. (2014). Weed management on lentil with post emergence herbicides. Indian Journal of Weed Science., 46(2) : 187-189.

xvi. Hamada, A.A. 1988. Weed competition in irrigated groundnut variety ashford, in the Rahad Scheme, Sudan. Veterinarmedian, 26: 25-31.

xvii. Page, W. W., Busolo-Bulafu, C. M., Vander, P. J. A. and Chancellor, T. C. B. (2002). Recommended Groundnut Production Practices for Small Holder Farmers in Uganda. The University of Greenwich. London, pp. 3-4.

xviii. Sumathi, P and Muralidharan, V (2007) . Character association and path coefficient analysis in confectionery type groundnut (Arachishypogaea L.). Madras Agriculture Journal 94 (1-6): 105-109.

xix. Singh V .B. and Giri G. (2001). Influence of intercropping and weed control measures on dry matter accumulation and nutrient uptake by sunflower and groundnut and their effect on succeeding maize. Indian Journal of Agronomy, 46(1): 50-55

xx. Sankaranarayana, V., Venkataraman, P., Reddy, V. S., Fathima, P.S., Raju, G.T. T. and Muniyappa, T. V.2000. Studies on integrated weed management in Kharif groundnut. Mysore Journal of Agricultural Science 34: 21-26.

xxi. Ashutosh K., Soma G., Sheetal R. S and Pradhan K (2016). Genetic Variability, Correlation Coefficient and Path Coefficient Analysis for Yield and Component Traits in Groundnut. Indian Journal of Ecology 43 (2): 85-89

xxii. Babariya C.A and Dobariya K.L (2012) . Correlation coefficient and path coefficient analysis for yield components in groundnut (Arachis hypogaea L.). Electronic Journal of Plant Breeding 3(3): 932-938

xxiii. Dhaliwal G.P.S., Nagada A.K and Mittal V. P (2010). Intertrait associations and path analysis studies in groundnut (Arachishypogaea L.). Crop improvement 37(1): 57-60. 